\title{
Wide-field optical model of the human eye with asymmetrically tilted and decentered lens that reproduces measured ocular aberrations: supplementary material
}

\author{
James Polans, ${ }^{1,{ }^{*}}$ Bart Jaeken, ${ }^{2}$ Ryan P. McNabb, ${ }^{3,1}$ Pablo Artal, ${ }^{2}$ Joseph A. \\ IZATT, ${ }^{1,3}$
}

${ }^{1}$ Department of Biomedical Engineering and Fitzpatrick Institute of Photonics, Duke University, Durham, North Carolina 27708, USA

${ }^{2}$ Laboratorio de Óptica, Universidad de Murcia, Campus de Espinardo (Edificio 34), 30100 Murcia, Spain

${ }^{3}$ Department of Ophthalmology, Duke University Medical Center, Durham, North Carolina 27710, USA

*Corresponding author: james.polans@duke.edu

Published 30 January 2015

\begin{abstract}
This document provides supplementary information to "Wide-field optical model of the human eye with asymmetrically tilted and decentered lens that reproduces measured ocular aberrations," http://dx.doi.org/10.1364/optica.2.000124. Further detail is provided on the optimization process used to generate the eye model. A representative subset of the full merit function is included to illustrate the types of constraints and relative weights of the operands used during optimization. Discussion of the limitations of GRIN surface representation in optical design software is provided. Additional figures compare the measured wavefront data along the vertical meridian to those values reproduced in the newly proposed and existing eye models. Detail and discussion are provided on the wavefront phase profile of the model eye in the pupil plane. (c) 20150 ptical Society of America
\end{abstract}

http://dx.doi.org/10.1364/optica.2.000124.s001

\section{Eye Model Optimization Merit Function}

The reverse building optimization technique used to create the proposed eye model is depicted schematically in Fig. S1. The model was optimized in Zemax optical design software using a custom merit function (Table S1) that incorporated information about anatomical boundary constraints, GRIN lens properties, and measured wavefront aberration data.

Anatomical boundary constraints, derived from multiple sources and compiled more succinctly in $[1,2]$, limited the range of variation of anatomical features, such as the radii of curvature, thicknesses, and conic constants. Boundary constraints were divided qualitatively into "hard" and "soft" limits according to their weights within the merit function. Hard limits, which constrained the associated properties with very high weight, were used for all surface properties except for those pertaining to the lens. The hard limits forced the parameters into a desired target range without any preference for particular values within that range. Soft limits, which penalized variation outside the target range with much lower weight, were used for the anterior and posterior lens surfaces because anatomical values reported for the lens [1], including radius of curvature, thickness, and conic constants, often included simplifying assumptions, only described the lens over a small field-of-view, or were not measured in vivo. The maximum, minimum, and range of the index of the lens were constrained using hard limits based on the values derived in [3]. Tilt and decentration of the lens were not constrained in the merit function, but rather, they were allowed to vary freely.

Measured ocular aberration data as a function of retinal eccentricity was incorporated into the merit function as soft constraints with target values (as opposed to the target ranges used for the anatomical constraints), where the target values were the mean aberration magnitudes derived from our measured data sets (101 eyes along the horizontal meridian [4] and 10 eyes along the vertical meridian). The first 15 Zernike terms (through $4^{\text {th }}$ 
order), excluding piston, tip, and tilt, were inserted into the merit function with soft limits from $-40^{\circ}$ to $+40^{\circ}$ in $10^{\circ}$ steps along the horizontal meridian and $-20^{\circ}$ to $+20^{\circ}$ in $10^{\circ}$ steps along the vertical meridian. The values under $10^{\circ}$ from the on-axis $\left(0^{\circ}\right)$ point were given 10x greater merit function weight than the values in the periphery. Also, since there was twice the number of horizontal to vertical constraints, the vertical operands were given twice the weight.

The complete optimization merit function contained 201 total operands. Table S1 includes the complete set of anatomical boundary constraints and GRIN lens properties, but only a representative subset of the wavefront constraints. For brevity, the full set of wavefront constraints is listed for one angle $\left(40^{\circ}\right.$ nasal); additionally, the relative weights (but not the specific values) are listed for the Zernike terms (Z3-Z14) of the other measured angles.

Optical dispersion parameters for the cornea, aqueous humor, lens, and vitreous humor were based on the findings of Atchison and Smith [5] (Table 2). The chromatic response of the GRIN lens (Table 3) was implemented using the information provided in [6], which examined specifically the chromatic aberrations across a wide field of view for human eyes. The dispersion parameters were hard set in the glass type category of Zemax, and they were not allowed to vary with the optimization routine.

Hammer optimization, which is a global optimization feature in Zemax, was used to reproduce the measured Zernike aberrations while keeping the anatomical parameters within their boundary limits. Note that all of the values listed in Table 1 were allowed to vary simultaneously during global optimization. Hammer optimization was allowed to run for over 72 hours on a $3.20 \mathrm{GHz}$ Intel Core i7 processor with 12 cores, which accounts for approximately 2.5 million variations of the system. Therefore, we posit that our optimization technique reached a unique solution for a specific set of weighted operands.

While a variety of similar eye models could be generated by varying the relative weights of the different operands, we settled on the proposed model weights because the result matched well the measured data throughout the field of view, including those meridians not constrained directly in the merit function (Figure 4). If a specific feature, such as corneal biometry or peripheral defocus, needed to be constrained with greater precision, this could be achieved through adjustment of the associated merit function weights.

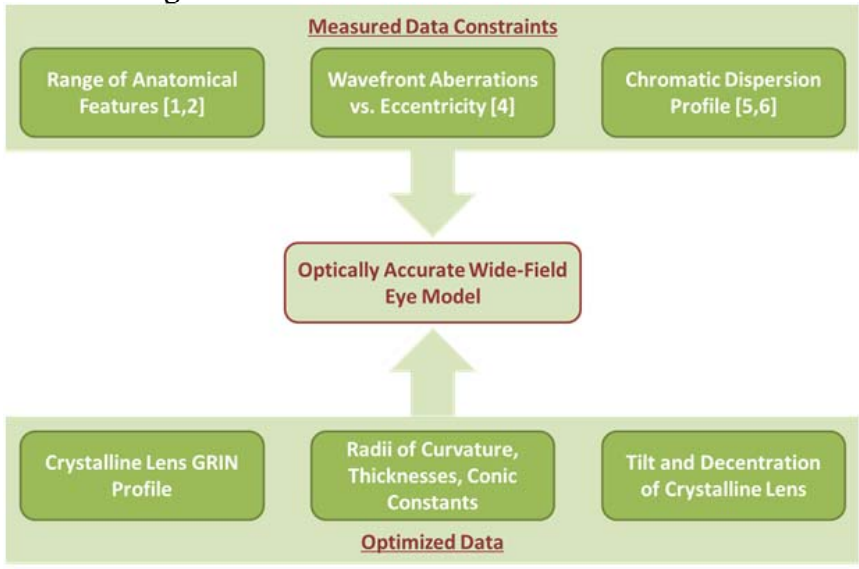

Fig. S1. Flow-diagram illustrating the eye model optimization process. The design method implemented a combination of experimentally measured data and raytracing optimization.

\section{GRIN Surfaces in Zemax}

In Zemax optical design software, GRIN lenses are defined as a piecewise series of infinitely thin refractive index plates where light does not refract between adjacent plates. The spatial distribution of refractive index is calculated only at a plate, and after refracting from a plate, each ray becomes associated with a refractive index until the next plate, regardless of whether the ray changes height or direction. Therefore, in order to reverse a GRINbased lens model, the refractive index in the reverse direction would need to be a function of both height and angle or the spacing of the index plates needs to be small. However, Zemax does not include surfaces that allow for refractive index to be defined as a function of both height and angle.

The $\Delta \mathrm{T}$ value from Table 1 corresponds to the axial sampling of the GRIN. While a ray trace of a highly sampled GRIN $(\Delta \mathrm{T}=0.010$ $\mathrm{mm}$ ) would more accurately simulate a continuous gradient, our reverse building process arrived at a large $\Delta \mathrm{T}$ value in order to match best the measured aberration data. Since there were limited gradient profiles available in Zemax and gradient 5 was the only profile that supported chromatic dispersion, we believe that our results could be improved even further with a more realistic axial GRIN profile. This treatment of GRIN profiles may help to explain why we obtained a slightly larger $\Delta \mathrm{T}$ and $\Delta \mathrm{n}(0.056)$ than expected (0.050) [3]. Previous works have not specified their chosen value of $\Delta \mathrm{T}$, and it is possible that others may have used a course sampling value too (the default value is $1 \mathrm{~mm}$ ). Thus, we believe caution should also be used in reversing prior eye models as well.

\section{Discussion of Wavefront Phase Profile}

Figure S4 demonstrates that the wavefront of the new eye model closely resembles that of the measured data. This implies that the optical path length of any individual ray across the entire pupil stop correctly mimics the optical path length differences in the average human eye. This feature has important implications for many common ophthalmic imaging systems, such as optical coherence tomography $[7,8]$, which incorporates interferometric optical depth ranging. Knowing the optical path length of an individual ray in the pupil at a given angle of incidence potentially could allow aberration corrected images to be obtained without the use of adaptive optics $[9,10]$. Computationally reconstructing aberrated images has been shown to be successful in examples such as deconvolution imaging [11-14], digital refocusing [15, 16] and synthetic aperture microscopy $[17,18]$.

\section{Additional Figures}

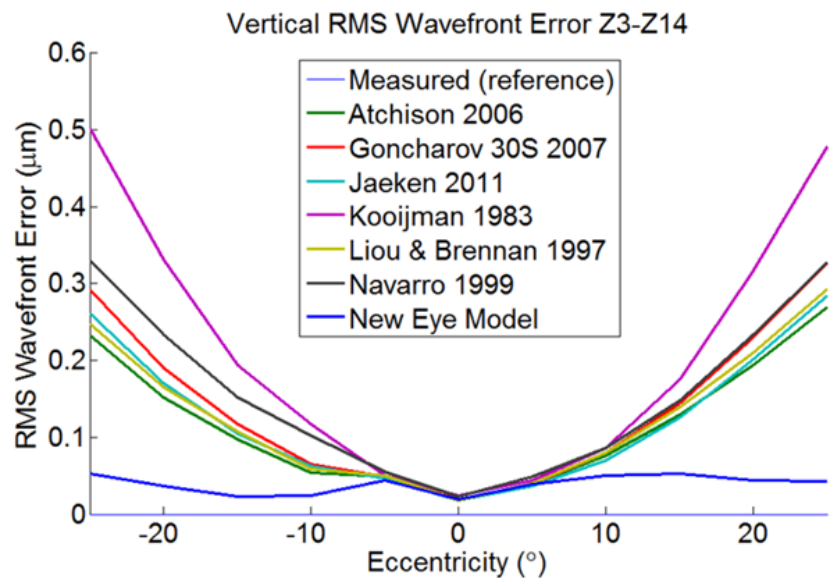

Fig. S2. RMS wavefront error of the various eye models as compared to the measured wavefront data set along the vertical meridian. The on-axis $\left(0^{\circ}\right)$ defocus magnitude for each eye model was subtracted from the defocus value of the other eccentricities in order to illustrate more clearly the variation in RMS 
wavefront error with retinal eccentricity. The standard deviation of the measured data set was $\sim 0.093 \mu \mathrm{m}$.
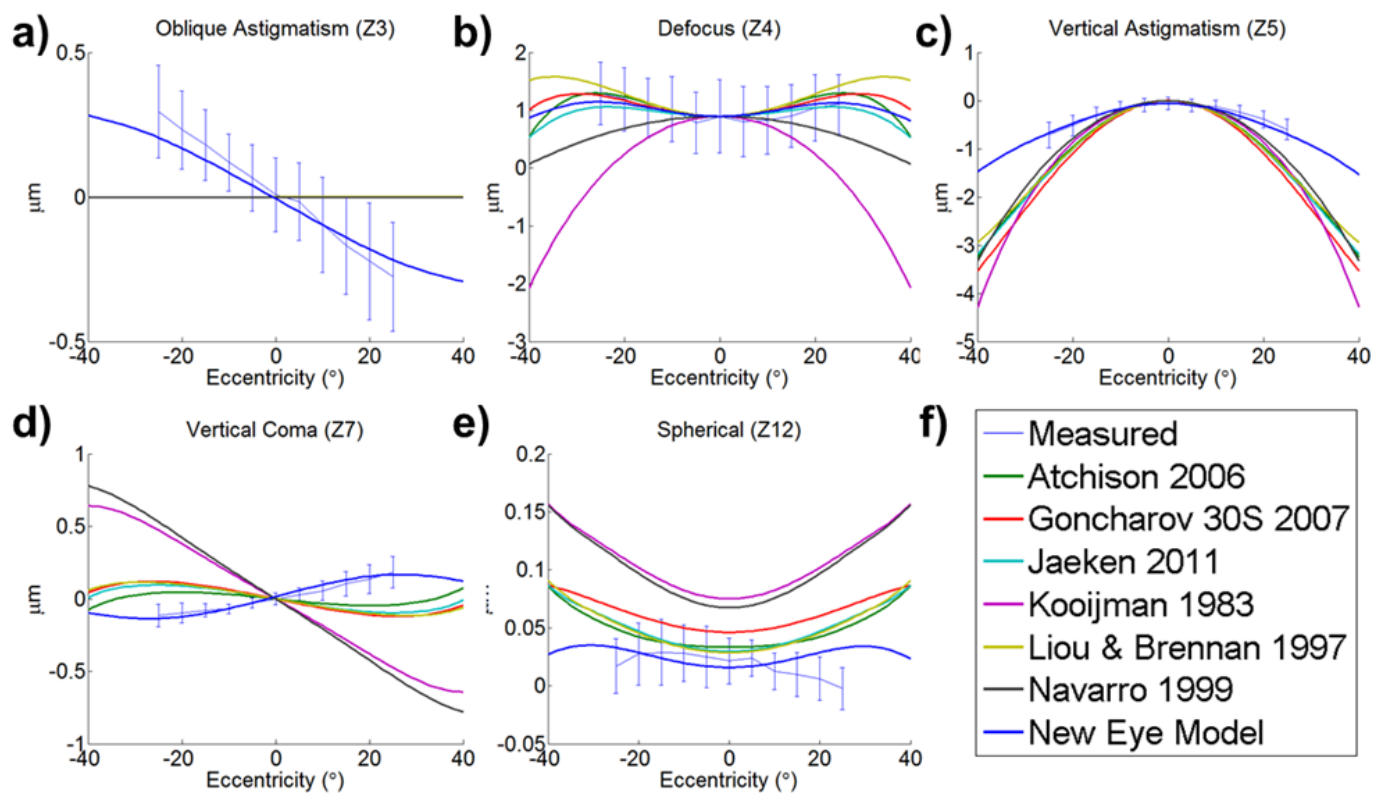

Fig. S3. Comparison of the same metrics as Fig. 3, but along the vertical meridian. The measured data in this figure corresponds to the vertical data taken from the supplementary group consisting of 10 eyes. The most significant aberration terms in the vertical direction were oblique astigmatism (a), defocus (b), vertical astigmatism (c), vertical coma (d), and spherical aberration (e). Like many of the other figures, the on-axis $\left(0^{\circ}\right)$ defocus magnitude for each eye model was subtracted from the defocus value of the other eccentricities in order to emphasize the variation in RMS wavefront error with retinal eccentricity. The error bars correspond to the standard deviation in the measured data set for the group of 10 individuals.

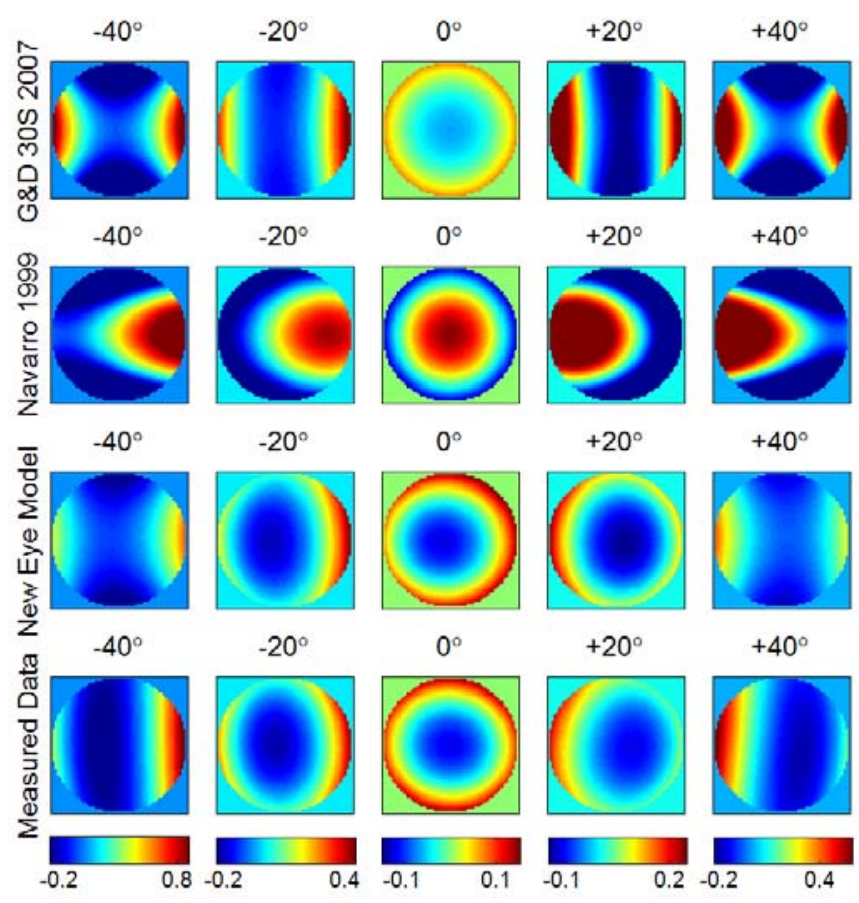

Fig. S4. Wavefront phase profile in the pupil plane for two other wide-field schematic eyes (Navarro [19], G\&D 30S [20]), our proposed eye, and the average of the measured data set [4]. The phase profiles displayed represent the phase profile at various retinal eccentricities. The profiles are presented in units of wavelengths for $780 \mathrm{~nm}$ light. 


\section{References}

1. M. M. Kong, Z. S. Gao, X. H. Li, S. H. Ding, X. M. Qu, and M. Q. Yu, "A generic eye model by reverse building based on Chinese population," Opt Express 17, 13283-13297 (2009).

2. J. J. Rozema, D. A. Atchison, and M. J. Tassignon, "Statistical Eye Model for Normal Eyes," Invest Ophth Vis Sci 52, 4525-4533 (2011).

3. C. E. Jones, D. A. Atchison, R. Meder, and J. M. Pope, "Refractive index distribution and optical properties of the isolated human lens measured using magnetic resonance imaging (MRI)," Vision Res 45, 2352-2366 (2005).

4. B. Jaeken and P. Artal, "Optical Quality of Emmetropic and Myopic Eyes in the Periphery Measured with High-Angular Resolution," Invest Ophth Vis Sci 53, 3405-3413 (2012).

5. D. A. Atchison and G. Smith, "Chromatic dispersions of the ocular media of human eyes," J Opt Soc Am A 22, 29-37 (2005).

6. B. Jaeken, L. Lundstrom, and P. Artal, "Peripheral aberrations in the human eye for different wavelengths: off-axis chromatic aberration," J Opt Soc Am A 28, 1871-1879 (2011).

7. E. A. Swanson, J. A. Izatt, M. R. Hee, D. Huang, C. P. Lin, J. S. Schuman, C. A. Puliafito, and J. G. Fujimoto, "In-Vivo Retinal Imaging by Optical Coherence Tomography," Opt Lett 18, 1864-1866 (1993).

8. D. Huang, E. A. Swanson, C. P. Lin, J. S. Schuman, W. G. Stinson, W. Chang, M. R. Hee, T. Flotte, K. Gregory, C. A. Puliafito, and J. G. Fujimoto, "Optical Coherence Tomography," Science 254, 1178-1181 (1991).

9. J. Z. Liang, D. R. Williams, and D. T. Miller, "Supernormal vision and highresolution retinal imaging through adaptive optics," J Opt Soc Am A 14, 2884-2892 (1997).
10. A. Roorda, F. Romero-Borja, W. J. Donnelly, H. Queener, T. J. Hebert, and M. C. W. Campbell, "Adaptive optics scanning laser ophthalmoscopy," Opt Express 10, 405-412 (2002).

11. V. Nourrit, B. Vohnsen, and P. Artal, "Blind deconvolution for highresolution confocal scanning laser ophthalmoscopy," J Opt a-Pure Appl Op 7, 585-592 (2005).

12. D. Catlin and C. Dainty, "High-resolution imaging of the human retina with a Fourier deconvolution technique," J Opt Soc Am A 19, 1515-1523 (2002).

13. J. C. Christou, A. Roorda, and D. R. Williams, "Deconvolution of adaptive optics retinal images," J Opt Soc Am A 21, 1393-1401 (2004).

14. G. R. Ayers and J. C. Dainty, "Iterative Blind Deconvolution Method and Its Applications," Opt Lett 13, 547-549 (1988).

15. J. Jang, J. Lim, H. Yu, H. Choi, J. Ha, J. H. Park, W. Y. Oh, W. Jang, S. Lee, and Y. Park, "Complex wavefront shaping for optimal depth-selective focusing in optical coherence tomography," Opt Express 21, 2890-2902 (2013).

16. G. Z. Liu, Z. W. Zhi, and R. K. K. Wang, "Digital focusing of OCT images based on scalar diffraction theory and information entropy," Biomed Opt Express 3, 2774-2783 (2012).

17. T. S. Ralston, D. L. Marks, P. S. Carney, and S. A. Boppart, "Interferometric synthetic aperture microscopy," Nat Phys 3, 129-134 (2007).

18. X. D. Chen, Q. Li, Y. Lei, Y. Wang, and D. Y. Yu, "Sdoct Image Reconstruction by Interferometric Synthetic Aperture Microscopy," J Innov Opt Heal Sci 3, 17-23 (2010).

19. I. Escudero-Sanz and R. Navarro, "Off-axis aberrations of a wide-angle schematic eye model," J Opt Soc Am A 16, 1881-1891 (1999).

20. A. V. Goncharov and C. Dainty, "Wide-field schematic eye models with gradient-index lens," J Opt Soc Am A 24, 2157-2174 (2007). 
Table S1. Subset of Optimization Merit Function

\begin{tabular}{|c|c|c|c|c|c|}
\hline Surface/Angle & Property & Type & Target & Weight & Description \\
\hline \multicolumn{6}{|c|}{ Anatomical Boundary Constraints } \\
\hline Anterior Cornea & Radius of Curvature & Greater than & $7.56 \mathrm{~mm}$ & $1,000,000$ & Hard limit, from $[1,2]$ \\
\hline Anterior Cornea & Radius of Curvature & Less than & $8.16 \mathrm{~mm}$ & $1,000,000$ & Hard limit, from $[1,2]$ \\
\hline Posterior Cornea & Radius of Curvature & Greater than & $6.05 \mathrm{~mm}$ & $1,000,000$ & Hard limit, from $[1,2]$ \\
\hline Posterior Cornea & Radius of Curvature & Less than & $6.86 \mathrm{~mm}$ & $1,000,000$ & Hard limit, from $[1,2]$ \\
\hline Anterior Lens & Radius of Curvature & Greater than & $9.03 \mathrm{~mm}$ & 1 & Soft limit, from $[1,2]$ \\
\hline Anterior Lens & Radius of Curvature & Less than & $12.41 \mathrm{~mm}$ & 1 & Soft limit, from $[1,2]$ \\
\hline Posterior Lens & Radius of Curvature & Greater than & $5.75 \mathrm{~mm}$ & 1 & Soft limit, from $[1,2]$ \\
\hline Posterior Lens & Radius of Curvature & Less than & $7.71 \mathrm{~mm}$ & 1 & Soft limit, from $[1,2]$ \\
\hline Retina & Radius of Curvature & Greater than & $11.00 \mathrm{~mm}$ & $1,000,000$ & Hard limit, from $[1,2]$ \\
\hline Retina & Radius of Curvature & Less than & $13.10 \mathrm{~mm}$ & $1,000,000$ & Hard limit, from $[1,2]$ \\
\hline Anterior Cornea & Conic Constant & Greater than & -0.30 & 1 & Soft limit, from $[1,2]$ \\
\hline Anterior Cornea & Conic Constant & Less than & 0.00 & 1 & Soft limit, from $[1,2]$ \\
\hline Posterior Cornea & Conic Constant & Greater than & -0.30 & 1 & Soft limit, from $[1,2]$ \\
\hline Posterior Cornea & Conic Constant & Less than & 0.16 & 1 & Soft limit, from $[1,2]$ \\
\hline Anterior Lens & Conic Constant & Greater than & -3.20 & 1 & Soft limit, from $[1,2]$ \\
\hline Anterior Lens & Conic Constant & Less than & 0.25 & 1 & Soft limit, from $[1,2]$ \\
\hline Posterior Lens & Conic Constant & Greater than & -1.00 & 1 & Soft limit, from $[1,2]$ \\
\hline Posterior Lens & Conic Constant & Less than & 1.00 & 1 & Soft limit, from $[1,2]$ \\
\hline Cornea & Thickness & Greater than & $0.50 \mathrm{~mm}$ & $1,000,000$ & Hard limit, from $[1,2]$ \\
\hline Cornea & Thickness & Less than & $0.56 \mathrm{~mm}$ & $1,000,000$ & Hard limit, from $[1,2]$ \\
\hline Anterior Chamber & Thickness & Greater than & $2.91 \mathrm{~mm}$ & $1,000,000$ & Hard limit, from $[1,2]$ \\
\hline Anterior Chamber & Thickness & Less than & $3.65 \mathrm{~mm}$ & $1,000,000$ & Hard limit, from $[1,2]$ \\
\hline Lens & Thickness & Greater than & $3.46 \mathrm{~mm}$ & 1 & Soft limit, from $[1,2]$ \\
\hline Lens & Thickness & Less than & $4.06 \mathrm{~mm}$ & 1 & Soft limit, from $[1,2]$ \\
\hline Posterior Chamber & Thickness & Greater than & $16.00 \mathrm{~mm}$ & $1,000,000$ & Hard limit, from $[1,2]$ \\
\hline Posterior Chamber & Thickness & Less than & $17.00 \mathrm{~mm}$ & $1,000,000$ & Hard limit, from $[1,2]$ \\
\hline \multicolumn{6}{|c|}{ GRIN Lens Properties } \\
\hline GRIN of Lens & Index Min & Greater than & $1.350 \mathrm{RIU}$ & $1,000,000$ & Hard limit, from [3] \\
\hline GRIN of Lens & Index Max & Less than & $1.420 \mathrm{RIU}$ & $1,000,000$ & Hard limit, from [3] \\
\hline GRIN of Lens & Index Change & Less than & 0.065 RIU & $1,000,000$ & Hard limit, from [3] \\
\hline \multicolumn{6}{|c|}{ Measured Wavefront Aberration Data } \\
\hline $40^{\circ} \mathrm{Nasal}$ & Z3 (Ob. Astig.) & Equal & $0.167 \lambda$ & 1 & Soft limit, from [4] \\
\hline $40^{\circ}$ Nasal & Z4 (Defocus) & Equal & $0.703 \lambda$ & 1 & Soft limit, from [4] \\
\hline $40^{\circ}$ Nasal & Z5 (Vert. Astig.) & Equal & $1.138 \lambda$ & 1 & Soft limit, from [4] \\
\hline $40^{\circ} \mathrm{Nasal}$ & Z6 (Vert. Trefoil) & Equal & $0.063 \lambda$ & 1 & Soft limit, from [4] \\
\hline $40^{\circ} \mathrm{Nasal}$ & Z7 (Vert. Coma) & Equal & $0.052 \lambda$ & 1 & Soft limit, from [4] \\
\hline $40^{\circ} \mathrm{Nasal}$ & Z8 (H. Coma) & Equal & $0.406 \lambda$ & 1 & Soft limit, from [4] \\
\hline $40^{\circ} \mathrm{Nasal}$ & Z9 (Ob. Trefoil) & Equal & $0.110 \lambda$ & 1 & Soft limit, from [4] \\
\hline $40^{\circ} \mathrm{Nasal}$ & Z10 (Ob. Quadrafoil) & Equal & $0.007 \lambda$ & 1 & Soft limit, from [4] \\
\hline $40^{\circ} \mathrm{Nasal}$ & Z11 (Ob. 2nd Astig.) & Equal & $0.001 \lambda$ & 1 & Soft limit, from [4] \\
\hline $40^{\circ} \mathrm{Nasal}$ & Z12 (Spherical) & Equal & $0.031 \lambda$ & 1 & Soft limit, from [4] \\
\hline $40^{\circ} \mathrm{Nasal}$ & Z13 (Vert. 2 ${ }^{\text {nd }}$ Astig.) & Equal & $0.020 \lambda$ & 1 & Soft limit, from [4] \\
\hline $40^{\circ} \mathrm{Nasal}$ & Z14 (Vert. Quadrafoil) & Equal & $0.012 \lambda$ & 1 & Soft limit, from [4] \\
\hline $30^{\circ}$ Nasal & $\mathrm{Z3}-\mathrm{Z14}$ & Equal & -- & 1 & Soft limit, from [4] \\
\hline $20^{\circ} \mathrm{Nasal}$ & $\mathrm{Z3}-\mathrm{Z14}$ & Equal & -- & 1 & Soft limit, from [4] \\
\hline $10^{\circ}$ Nasal & $\mathrm{Z3}-\mathrm{Z14}$ & Equal & -- & 10 & Soft limit, from [4] \\
\hline $0^{\circ}$ (on-axis) & $\mathrm{Z3}-\mathrm{Z14}$ & Equal & -- & 10 & Soft limit, from [4] \\
\hline $10^{\circ}$ Temporal & $\mathrm{Z3}-\mathrm{Z14}$ & Equal & -- & 10 & Soft limit, from [4] \\
\hline $20^{\circ}$ Temporal & $\mathrm{Z3}-\mathrm{Z14}$ & Equal & -- & 1 & Soft limit, from [4] \\
\hline $30^{\circ}$ Temporal & $\mathrm{Z3}-\mathrm{Z14}$ & Equal & -- & 1 & Soft limit, from [4] \\
\hline $40^{\circ}$ Temporal & $\mathrm{Z3}-\mathrm{Z14}$ & Equal & -- & 1 & Soft limit, from [4] \\
\hline $20^{\circ}$ Inferior & $\mathrm{Z3}-\mathrm{Z14}$ & Equal & -- & 2 & Soft limit, from new 2D data \\
\hline $10^{\circ}$ Inferior & $\mathrm{Z3}-\mathrm{Z14}$ & Equal & -- & 20 & Soft limit, from new 2D data \\
\hline $10^{\circ}$ Superior & $\mathrm{Z3}-\mathrm{Z14}$ & Equal & -- & 20 & Soft limit, from new 2D data \\
\hline $20^{\circ}$ Superior & $\mathrm{Z3}-\mathrm{Z14}$ & Equal & -- & 2 & Soft limit, from new 2D data \\
\hline
\end{tabular}

